\title{
La formación de la conciencia geográfica en el aula. Estudio de casos en educación secundaria en Chile ${ }^{1}$
}

\author{
The formation of the geographical consciousness in \\ the classroom. A case-study of the Chilean Secondary \\ Education
}

Evelyn Viviana Ortega Rocha² (10 y Joan Pagès Blanch ${ }^{3}$

\begin{abstract}
RESUMEN
En la actualidad, la enseñanza de la Geografía implica experiencias de aula que desafíen al estudiantado para la toma de decisiones. La conciencia geográfica es fundamental para que niños y jóvenes comprendan los cambios espaciales de la sociedad y actúen, desde las posibilidades que otorga la perspectiva geográfica, ante los problemas espaciales y ambientales relevantes de su realidad.

El objetivo de esta investigación es analizar las decisiones que toma el profesorado en sus clases para formar, o no, una conciencia geográfica crítica. También se presentan los ejes orientadores a partir de los cuales interpretamos las prácticas docentes. Se aplica una metodología mixta con predominio del enfoque cualitativo a partir del estudio de casos. Se realizaron entrevistas y observaciones de aula a cinco docentes chilenos del área de Historia y Ciencias Sociales.

Los resultados indican que el profesorado está formando principalmente una conciencia geográfica de carácter descriptivo - analítica porque mantiene una tradición de enseñanza memorística, se mueve en escalas locales y globales en una relación lineal, trata la temporalidad en un momento concreto o desde el pasado al presente, y el protagonismo lo tienen los territorios (en disputa, puntos estratégicos, lugares concretos), mientras que los hombres y las mujeres son invisibilizados.
\end{abstract}

Palabras claves: pensamiento geográfico, conciencia geográfica, didáctica de la geografía, profesorado.

Esta investigación fue posible gracias a la beca otorgada por la Agencia Nacional de Investigación (ANID) PFCHA / DOCTORADO BECAS CHILE / 2016 - 72170215 para realizar estudios de doctorado.

Estudiante de Doctorado, Departamento de Didáctica de la Lengua y la Literatura, y de las Ciencias Sociales, Universidad Autónoma de Barcelona. Correo electrónico: eveortega@gmail.com

+ Catedrático emérito, Departamento de Didáctica de la Lengua y la Literatura, y de las Ciencias Sociales, Universidad Autónoma de Barcelona. Reconocimiento póstumo al profesor Joan Pagès, quien participó en la discusión, reflexión, redacción y correcciones del presente artículo. Por su compromiso permanente de contribuir a la investigación en Didáctica, pero, sobre todo a la enseñanza de las Ciencias Sociales. 


\begin{abstract}
Currently, teaching Geography implies classroom experiences that challenge students to make important decisions. Geographic consciousness plays a pivotal role to children and youngsters to comprehend the spatial changes of society, and also, to act against spatial and environmental problems relevant to their realities. The latter said by the possibilities that are granted by a geographical perspective.

The aim of this case-study is to analyze whether teachers take into account the critical geographical consciousness formation when teaching. Also, the main themes, which are used to interpret the practice of teaching, are shown. The mixed method is used in this research; however, it is worth mentioning that there is a predominance of the qualitative method. In order to gather information, interviews and classroom observation from five Chilean teachers from History and social science were carried out.

Results show that teachers are forming students focusing on geographical consciousness. This focus is based upon a descriptive-analytic position given that it maintains a memory-teaching tradition, which variates from local and global scales. As well, this position considers the temporality of a specific moment, or another moment from past to present, and finally, it also considers the importance of the territories (in dispute, strategical points, specific places) whereas men and women are not addressed.
\end{abstract}

Key words: geographic thinking, geographic consciousness, didactic geography, teachers.

En Chile, el papel de la geografía en la enseñanza ha sido motivo de debate en los últimos años. El currículo se ha actualizado en 2009 y en 2013, incorporando entre sus ejes el desarrollo de un pensamiento geográfico. Sin embargo, este pensamiento se aborda de manera insuficiente en las propuestas curriculares.

Como es sabido, el currículo es un instrumento de naturaleza política, y como tal, cambia más rápido que la práctica de aula. Esta última obedece a cambios más complejos de interpretar ya que es una realidad viva (Pagès, 2009). Por ello, para cambiarla es fundamental conocerla a través de las representaciones, de las decisiones didácticas y de las acciones que toma el profesorado cuando programa y cuando enseña.

Las investigaciones sobre las prácticas docentes en Geografía plantean que el profesorado mantiene una lucha permanente con su herencia positivista y descriptiva que se refleja en su enseñanza (Miranda, 2012; Arenas, 2017). Por su parte, Ortega (2019) explica que el profesorado no se siente preparado ni disciplinar ni didácticamente para enseñar Geografía, y menos aún, para posicionarse en la formación de un pensamiento y una conciencia geográfica crítica.

En consecuencia, observamos, por un lado, un currículo que no aborda la comprensión de la realidad geográfica actual, sino que lo hace de manera parcializada y, por el otro, la falta de herramientas pedagógicas y didácticas en el profesorado para actuar en la transformación de ese currículo en la práctica.

Estos antecedentes nos llevan a considerar la necesidad de profundizar en el pensamiento geográfico y cómo éste permite a los jóvenes desarrollar una conciencia geográfica que les ayude a comprender los cambios espaciales de una sociedad y a actuar, desde las posibilidades que otorga la perspectiva geográfica, ante los problemas espaciales y ambientales relevantes de nuestro entorno. 
En síntesis, es necesario, y urgente, formar en los niños y en las niñas y en los y las jóvenes una conciencia geográfica que les permita comprender "la geograficidad del presente, la relatividad de los lugares y de las interpretaciones de los lugares habitados" (Laurín, 2001: 199).

\section{Marco teórico de la investigación}

\section{Enseñanza de la Geografía}

Según Benejam (1997a), existen cuatro tradiciones epistemológicas en el campo científico y en el campo de la enseñanza de las ciencias sociales -la tradición positivista, la tradición humanista o reconceptualista, la concepción crítica y la tradición postmodernista. Sin embargo, Tutiaux-Guillon (2008) considera que una lectura de la realidad educativa en términos de "paradigma pedagógico", es decir relacionando el saber, la enseñanza y el aprendizaje, permite reducir el análisis de la situación de la enseñanza de las ciencias sociales -y de la geografía- a dos paradigmas: el paradigma clásico -el positivista- y el paradigma alternativo -constructivista/crítico.

En nuestra opinión, el paradigma positivista se adscribe a una formación ciudadana basada en consolidar la relación entre territorio - paisaje - identidad y con ello, incentivar, incluso hoy, el nacionalismo patriótico. El segundo, en cambio, busca fortalecer una ciudadanía democrática con la intención de utilizar la geografía para interpretar el mundo actual, pensar y gestionar alternativas dirigidas a resolver problemas como el deterioro ambiental, la desigualdad, la injusticia social, el cambio climático, la sobrepoblación, entre otros.

Pagès (1998) planteó que "la enseñanza de las Ciencias Sociales, de la Geografía y de la Historia tal como está concebida suele tener poco impacto, aunque lo tiene, en la formación de la visión del mundo y en la práctica social del alumnado" (p. 8). Ello podría deberse, según analizó Tutiaux - Guillón (2006) para el caso francés, a que en la enseñanza de la historia o de la geografía en secundaria los contenidos continúan siendo cosificados y priman los conocimientos factuales y nocionales. Para Fernández (2008) el problema parece radicar en cómo se interpreta la función de la geografía en la escuela. Así, para dar cuenta de un mundo social, complejo y conflictivo, el contenido muchas veces se presenta para mostrar a los alumnos cómo es en realidad el mundo y denunciar las injusticias, en lugar de brindar herramientas y formar capacidades para comprender la dinámica social y participar más plenamente en ella.

Ante esta situación, autores como, Benejam (1992), Fien (1999), Laurín (2001), Merenne Schoumaker (2005), Souto (2007a), García (2013), Cavalcanti y De Souza (2015) proponen una enseñanza de la Geografía útil para que niños y niñas y jóvenes aprendan a pensar el espacio y a comprender los problemas sociales, culturales, ambientales y económicos existentes en el mundo. Y también, para actuar y desenvolverse en sus espacios, interpretando la realidad desde diferentes perspectivas y moviéndose a distintas escalas.

Enseñar una geografía comprometida implica experiencias de aula que desafíen y empoderen a los estudiantes para trabajar por la justicia social y la sostenibilidad ecológica. Los saberes geográficos no son saberes ideológicamente neutros, por tanto, cualquier actividad que se planteé debe tratar crítica y creativamente esa realidad con el fin de mejorarla (Fien, 1999). 
En consecuencia, las finalidades de la enseñanza de la geografía en la actualidad, siguiendo, entre otros, a Gurevich, Blanco, Fernández y Tobío, 1995; Le Roux, 1997; Benejam, 1997a; Merenne - Schoumaker, 2005; Callai, 2012; Araya, 2013; Arenas y Salinas, 2013; Arenas, Fernández y Pérez, 2016; Unión Geográfica Internacional (UGI), 2016 deberían centrarse en:

- Enseñar a pensar el espacio para favorecer una conciencia social crítica.

- Estudiar la organización del territorio, de las sociedades y su espacio (localizaciones, distribuciones, relaciones, cambios, permanencias y posibilidades).

- Favorecer la decodificación de la realidad espacial de las personas (alfabetización).

- Enseñar a gestionar diferentes herramientas de representación espacial (mapas, diagramas, gráficos, fotografías, etc.) que puedan actuar como mediadores en la toma de decisiones geográficas.

- Desarrollar un pensamiento crítico para vivir de forma sostenible, local y globalmente, y para que la gente actúe de forma coherente.

- Propiciar la comprensión de las interacciones entre el ser humano y el medio natural, social, político, económico, cultural.

- Preparar para la acción: circular, viajar, comprender la información de los medios de comunicación, ser un ciudadano responsable, consciente del medio ambiente.

El saber geográfico es útil no solo por estas finalidades sino también porque aporta conocimientos y habilidades imprescindibles en la planificación urbana, en la geopolítica y en la guerra, en la toma de decisiones económicas, en las políticas públicas que se gestionan en los territorios, en las propuestas sobre el medio ambiente, el cambio climático, etc.

\section{Conciencia Geográfica}

Entendemos la conciencia geográfica como parte de un proceso reflexivo individual y colectivo que se materializa en las acciones, es decir, en el desarrollo en los estudiantes y en la ciudadanía de un pensamiento para la toma de decisiones espaciales, decisiones que incluyen, implícita o explícitamente, valores, actitudes, compromiso ético y la memoria, además, por supuesto, de saberes. Estas acciones se basan en una visión de la geografía que construye posibilidades o alternativas y movilizan positivamente para el cambio (Gardner \& Lambert; 2006; Hicks, 2007; Hicks, 2014). La acción genera reflexión y pensamiento que provee de argumentos a la ciudadanía para nuevas acciones.

Para Klein y Laurín (1999) y Tituaux-Guillon (2003) dotar de una conciencia territorial activa a los ciudadanos y ciudadanas significa desarrollar su sensibilidad respecto a su lugar de pertenencia, y a su vez, llevarles a asumir un compromiso global frente a los problemas. Por su parte, Garrido (2005) entiende la conciencia como una categoría problematizadora que permite recuperar las posibilidades de actuación-transformación de la experiencia espacial. En consecuencia, la 
conciencia geográfica se adquiere a través de un conjunto de situaciones educativas de carácter interactivo (Laurín, 2001).

La formación de la conciencia geográfica, al igual que la de la conciencia histórica, es fundamental para la formación democrática de la ciudadanía (Santisteban, 2010). La conciencia geográfica nos interroga sobre nuestra realidad socio-espacial, y sobre su temporalidad, nos enfrenta a ella y nos conduce a tomar decisiones adecuadas a partir de un discurso geográfico sólido. Por lo tanto, la construcción de la conciencia geográfica en los alumnos de secundaria (también en los de primaria, y en los universitarios) fomenta competencias sociales y ciudadanas para la intervención social.

\section{Ejes orientadores para abordar la conciencia geográfica en el aula}

El análisis del desarrollo de la conciencia geográfica en el aula ha exigido la elaboración de una propuesta a partir de aquellos elementos que aparecen en la bibliografía, en los currículos de diferentes países, en las entrevistas a didactas del área y en las entrevistas al profesorado.

Partimos de las ideas de Freire (1980) sobre la dinámica de la conciencia. Aunque Freire no trata la conciencia geográfica como tal, la dinámica que ofrece es interesante para esta investigación porque examina el papel de la educación en el desarrollo de la conciencia que hace posible a los ciudadanos la autorreflexión sobre su tiempo y su espacio. Esta autorreflexión llevará a los niños y a los jóvenes a convertirse en actores y autores de su espacio geográfico.

Una conciencia geográfica crítica emerge de la práctica del pensamiento geográfico que permite a los ciudadanos situarse y comprender el espacio geográfico, un espacio geográfico construido social e históricamente (Lefebvre, 2013; Santos; 1990) para resolver problemas geográficos y ambientales.

Laurín (2001) entiende la conciencia geográfica como un elemento del pensamiento geográfico. En esta línea, en Ortega (2019) se presentan las aportaciones de diferentes didactas del área de las ciencias sociales que expresan que es posible desarrollar la conciencia geográfica a partir de conceptos como espacio y lugar, escala y conexión, proximidad y distancia y pensamiento relacional, fundamentales para el desarrollo del pensamiento geográfico. En el mismo texto, se expone que el desarrollo del pensamiento geográfico genera una conciencia geográfica, es decir, una conciencia de que las cosas, objetos, fenómenos con los que nos relacionamos en la vida diaria, directa o indirectamente. Y, se explica que la conciencia geográfica se construye a partir de la dialéctica entre el pensamiento geográfico que es producido por la ciencia y el pensamiento que es singular en cada persona, pero que se construye en una realidad social.

$Y$, en Ortega (2020) presentamos las concepciones de las y los docentes chilenos que participan en la investigación, rescatando el aporte de Marco que cree que el pensamiento lleva a la conciencia, porque al pensar se desarrollan habilidades de nivel superior que, en su conjunto, permitirán a los estudiantes desarrollar una conciencia geográfica.

En nuestra investigación entendemos la conciencia geográfica como el desarrollo máximo del pensamiento geográfico desde una perspectiva crítica y proyectiva, es decir, que no se queda en 
la crítica, sino que es capaz de generar alternativas para la solución de los problemas geográficos y ambientales. En palabras de Pagès (2009: 152), "educar ciudadanos comprometidos que sepan lo que están haciendo, y lo hagan junto a los demás, para construir un mundo mucho más igualitario que el que tenemos actualmente."

En la figura $\mathrm{N}^{\circ} 1$ presentamos la propuesta que hemos elaborado para abordar la conciencia geográfica en el aula, centrándonos en unos problemas geográficos, unas habilidades de razonamiento geográfico y unos elementos claves (multiescalaridad, temporalidad y protagonistas) que, según las decisiones profesionales del profesorado, han de llevar al alumnado a un nivel de conciencia geográfica crítica.

Figura $\mathrm{N}^{\circ} 1$.

Estructura conceptual para abordar la conciencia geográfica en el aula

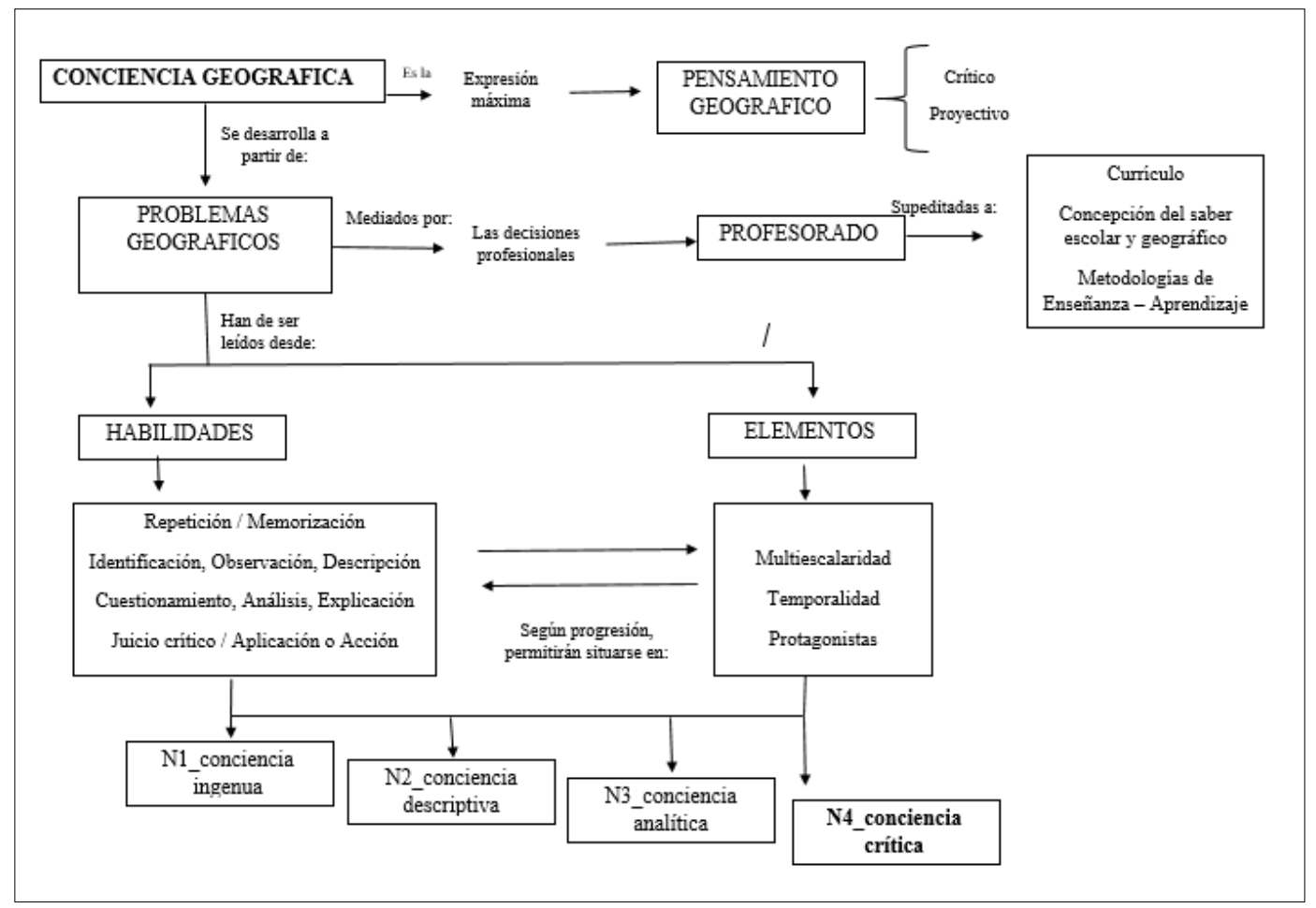

Fuente: Elaboración propia.

En el desarrollo de la conciencia geográfica intervienen los contenidos planteados en el currículo, pero mediados por las decisiones profesionales del profesorado. Además, si estos contenidos se centran en problemas, y en metodologías cooperativas e interactivas, el desarrollo de la conciencia geográfica del alumnado alcanzará con mayor facilidad y rapidez el último nivel de desarrollo, la conciencia crítico-reflexiva.

A partir de las ideas anteriores, de la concepción de conciencia de Freire, de las habilidades de razonamiento geográfico propuestas por Merenne - Schoumaker (2005) y de tres elementos claves en la enseñanza de la geografía (multiescalaridad, temporalidad y protagonistas) hemos 
establecido cuatro niveles de progreso de la conciencia: la no reflexiva - ingenua (Nivel 1), la descriptiva (Nivel 2), la analítica (Nivel 3) y la crítica (Nivel 4). La finalidad de la enseñanza de la geografía debería consistir en conducir a los estudiantes a desarrollar una conciencia geográfica crítica que les permita analizar las distintas alternativas y tomar decisiones geográficas adecuadas.

En consecuencia, para formar una conciencia geográfica, los problemas geográficos han de ser leídos desde las habilidades de razonamiento y desde el prisma de la multiescalaridad, la temporalidad y los protagonistas porque las interacciones que se producen en el espacio geográfico se desarrollan a diferentes escalas (local, regional, global, glocal), están situadas en diferentes temporalidades (pasado, presente y futuro) y son protagonizadas por hombres y mujeres (niños, niñas, jóvenes, adultos, ancianos y ancianas) que viven, actúan e intervienen en los territorios.

En el cuadro $\mathrm{N}^{0} 1$ se presenta la matriz de análisis para la formación de la conciencia geográfica en el aula. Esta tabla contempla las relaciones entre los niveles de conciencia geográfica definidos, las habilidades de razonamiento que se deberían desarrollar en cada nivel para alcanzar una conciencia geográfica crítica y unos indicadores (multiescalaridad, temporalidad y protagonistas) que permitirán leer los problemas geográficos propuestos por el profesorado en sus clases.

El N1 -conciencia geográfica no reflexiva- ingenua se centra exclusivamente en un aprendizaje memorístico y repetitivo de los contenidos geográficos. Las interacciones se focalizan en informaciones, fechas, lugares, símbolos, definiciones, etc. En este nivel se prioriza situar, localizar e individualizar territorialmente los hechos geográficos (Higueras, 2003). Se responde a la pregunta ¿Dónde están las cosas?

El N2 -conciencia geográfica descriptiva- se interesa por responder a la pregunta ¿qué son las cosas? Se centra en la observación, identificación y descripción. Se identifican las representaciones espaciales entendiéndolas como imágenes de la realidad que asumen conocimientos, signos, códigos y símbolos espaciales preestablecidos (Merenne - Schoumaker, 2005). Un segundo proceso cognitivo a desarrollar es la descripción que ha sido utilizada en la propuesta de Fien (1992), en los trabajos de Benejam (1997b; 2002) y en las pautas de análisis geográfico explicadas por Higueras (2003). La descripción consiste en relatar las características de los objetos, hechos, procesos o fenómenos geográficos estudiados y las circunstancias espacio-temporales que los rodean (Higueras, 2003). La descripción se apoya en la observación, la clasificación y la comparación y actúa como mediadora de la explicación.

El N3 -conciencia geográfica analítica- se interesa por saber por qué las cosas son como son. Se centra en el desarrollo de tres habilidades: el cuestionamiento, el análisis y la explicación. Para Benejam (1997b), las habilidades de cuestionamiento, análisis y explicación se orientan a la comprensión de las personas, de los grupos humanos, los hechos y los fenómenos. La comprensión del mundo implica la capacidad de establecer relaciones entre las informaciones para analizar las causas y consecuencias de determinados hechos y problemas.

El N4 - conciencia crítica responde a la pregunta ¿Qué hacemos?, y ¿Qué podríamos hacer? En este nivel el conocimiento alcanzado sobre determinado problema geográfico permite a los ciudadanos y ciudadanas analizar las distintas alternativas y optar por aquella que considera más pertinente. $Y$ sus decisiones se revierten en acciones concretas. En este nivel los y las alumnas 
son capaces de diseñar un proyecto para la solución de un problema, llevarlo a la práctica y valorar sus resultados y sus consecuencias inmediatas. Además, pueden emitir juicios de valor razonados sobre los problemas existentes en el mundo y las soluciones que se les están dando.

\section{Cuadro $N^{\circ} 1$.}

Matriz para el análisis de la formación de la conciencia geográfica en el aula

\begin{tabular}{|c|c|c|c|c|}
\hline $\begin{array}{l}\text { Categorías / } \\
\text { Indicadores }\end{array}$ & $\begin{array}{l}\text { N1_No } \\
\text { reflexiva - } \\
\text { ingenua }\end{array}$ & N2_descriptiva & N3_analítica & N4_crítica \\
\hline \multirow[t]{2}{*}{ Habilidades } & $\begin{array}{c}\text { Repetición } \\
\text { Memorización }\end{array}$ & $\begin{array}{l}\text { Observación } \\
\text { Identificación } \\
\text { Descripción }\end{array}$ & $\begin{array}{c}\text { Cuestionamien- } \\
\text { to } \\
\text { Análisis } \\
\text { Explicación }\end{array}$ & $\begin{array}{l}\text { Aplicación o acción } \\
\text { Juicio crítico } \\
\text { Diseño de alternativas }\end{array}$ \\
\hline & $\begin{array}{l}\text { ¿Dónde están } \\
\text { las cosas? }\end{array}$ & $\begin{array}{c}\text { ¿Qué son las } \\
\text { cosas? }\end{array}$ & $\begin{array}{c}\text { ¿Por qué las } \\
\text { cosas son como } \\
\text { son? }\end{array}$ & $\begin{array}{c}\text { ¿Qué hacemos? } \\
\text { ¿Qué podríamos } \\
\text { hacer? }\end{array}$ \\
\hline \multicolumn{5}{|c|}{ PROBLEMAS GEOGRÁFICOS Y AMBIENTALES } \\
\hline Multiescalaridad & \begin{tabular}{|c|} 
Se localizan en \\
puntos geográ- \\
ficos específi- \\
cos (ciudades, \\
países, ríos, \\
montañas, \\
parques, etc.) \\
según el conte- \\
nido. \\
\end{tabular} & $\begin{array}{l}\text { Se consideran } \\
\text { escalas locales } \\
\text { y/o globales } \\
\text { para tratar los } \\
\text { problemas } \\
\text { geográficos (re- } \\
\text { lación lineal). }\end{array}$ & \begin{tabular}{|c|} 
Se consideran \\
escalas locales y \\
escalas globa- \\
les para tratar \\
los problemas \\
geográficos (re- \\
lación concén- \\
trica).
\end{tabular} & $\begin{array}{l}\text { Se consideran múlti- } \\
\text { ples esferas espacia- } \\
\text { les, tanto locales como } \\
\text { globales para tratar los } \\
\text { problemas geográficos } \\
\text { (relación dialéctica). }\end{array}$ \\
\hline Temporalidad & \begin{tabular}{|c|} 
Se sitúan los \\
elementos geo- \\
gráficos en un \\
marco temporal \\
concreto (siglo, \\
año, mes, día, \\
primavera, \\
otoño, etc).
\end{tabular} & $\begin{array}{l}\text { Se considera } \\
\text { el cambio o la } \\
\text { continuidad en } \\
\text { los fenómenos } \\
\text { geográficos. } \\
\text { Se analiza el } \\
\text { territorio en una } \\
\text { realidad con- } \\
\text { creta (pasado o } \\
\text { presente). }\end{array}$ & $\begin{array}{c}\text { Se considera } \\
\text { el cambio y la } \\
\text { continuidad en } \\
\text { los fenómenos } \\
\text { geográficos. } \\
\text { Se compara el } \\
\text { territorio desde } \\
\text { una mirada dia- } \\
\text { crónica (pasa- } \\
\text { do - presente). } \\
\text { Se analizan las } \\
\text { causas y las } \\
\text { consecuencias. } \\
\end{array}$ & $\begin{array}{c}\text { Se considera el cambio } \\
\text { y la continuidad en los } \\
\text { fenómenos geográ- } \\
\text { ficos, estableciendo } \\
\text { relaciones. Se analiza } \\
\text { el territorio actual, } \\
\text { considerando una } \\
\text { mirada retrospectiva y } \\
\text { prospectiva. }\end{array}$ \\
\hline Protagonistas & \begin{tabular}{|c|} 
Los contenidos \\
geográficos \\
se centran en \\
los territorios, \\
invisibilizando a \\
los ciudadanos \\
y ciudadanas \\
que habitan en \\
ellos.
\end{tabular} & $\begin{array}{l}\text { Los contenidos } \\
\text { geográficos se } \\
\text { centran en los } \\
\text { territorios, pero } \\
\text { se visualiza la } \\
\text { acción de los } \\
\text { agentes de po- } \\
\text { der (Estado, Em- } \\
\text { presas, ONGs, } \\
\text { etc) en espacios } \\
\text { territoriales } \\
\text { específicos. }\end{array}$ & $\begin{array}{l}\text { Los contenidos } \\
\text { geográficos } \\
\text { se centran en } \\
\text { las relaciones } \\
\text { territorios - } \\
\text { ciudadanos (as), } \\
\text { donde hombres } \\
\text { y mujeres reales } \\
\text { participan, inte- } \\
\text { ractúan y crean } \\
\text { sus espacios } \\
\text { geográficos. }\end{array}$ & $\begin{array}{l}\text { Los contenidos geo- } \\
\text { gráficos se centran en } \\
\text { las relaciones terri- } \\
\text { torios - ciudadanos } \\
\text { (as). Los-hombres y las } \\
\text { mujeres se reconocen } \\
\text { como actores geográ- } \\
\text { ficos que reflexionan, } \\
\text { intervienen y toman } \\
\text { decisiones sobre los } \\
\text { problemas geográficos } \\
\text { que se presentan en su } \\
\text { vida cotidiana. }\end{array}$ \\
\hline
\end{tabular}

Fuente: Elaboración propia. 
Los elementos claves que actúan como indicadores para leer los problemas geográficos y ambientales son, como hemos señalado, la multiescalaridad, la temporalidad y los y las protagonistas.

La multiescalaridad refleja las complejas interacciones que atraviesan las sociedades actuales materializándose en lo que Castells denomina "sociedad en red" o "espacios de flujos" porque las interacciones disociadas espacialmente van adquiriendo un mayor peso en las experiencias de los ciudadanos y ciudadanas (Romero \& Goméz, 2008). La multiescalaridad es entendida como la concreción de múltiples esferas espaciales (Comes, 1998) o como la articulación dialéctica entre escalas locales y globales en la construcción de razonamientos espaciales complejos. Es decir, la multiescalaridad es una perspectiva espacial que supera el tratamiento dicotómico y excluyente de los fenómenos en su escala local o global, y viceversa, en una perspectiva meramente lineal (Cavalcanti, 2012).

Callai (2012) afirma que "o lugar não se explica por sí mesmo e o global só existe concretizado nos lugares" (p. 84). Es decir, tanto lo local como lo global conviven en una misma estructura espacial y son operadores espaciales que nos ayudan a comprender nuestras espacialidades y a cómo aprender a situarnos como seres glocales. Benejam (2011) afirma que para trabajar problemas geográficos la movilidad de la escala dependerá del objeto de estudio y, generalmente, intervienen diversos espacios geográficos.

La temporalidad se valora porque la práctica espacial de una sociedad deja huellas en el tiempo y esas huellas se descubren al descifrar su espacio. Las prácticas espaciales son: "complexas, fragmentadas, desiguais, diferenciadas, multiculturais, interculturais, desterritorializadas, reterritorializadas, organizadas em fluxos o redes, mediáticas e informatizadas" (Cavalcanti, 2008: 20). Implican estar inmersos en constantes decisiones, es decir, cada ciudadano o ciudadana se sitúa en un espacio donde se reconoce o se pierde, un espacio para disfrutar, controlar, gestionar o modificar (Lefebvre, 2013).

Stopani (1978) propone leer la realidad desde un análisis histórico - territorial para dar sentido a lo que se ha formado, transformado y conservado en el territorio por medio de generaciones de hombres y de mujeres, en su mayoría desconocidos, quienes, con su trabajo, han contribuido a una determinada configuración territorial.

Por su parte, Santos (1990) considera que el espacio se caracteriza, entre otras cosas, por la diferencia de edad entre los elementos que lo forman. Por lo tanto, cada lugar es el resultado de acciones multilaterales que se realizan en tiempos diferentes sobre cada uno, y en todos, los puntos de la superficie terrestre. El lugar asegura así la unidad de lo continuo y lo discontinuo, lo que a su vez posibilita su evolución y también le asegura una estructura concreta.

En síntesis, Stopani (1978), Bahbahani, Duquette, Sharpe y Tu Huynh (2016), Higueras (2003), Braudel (2002) y Mattozzi (2014) en sus escritos valoran los contrastes de los fenómenos geográficos en el tiempo y en el espacio. El currículo de Nueva Zelanda también considera la temporalidad como un elemento clave porque algunos de sus objetivos están orientados a la toma de decisiones sobre el futuro. Por ejemplo, el indicador del objetivo 7.2 plantea: "describe cómo las 
percepciones de las personas y las interacciones con sus entornos pueden cambiar el futuro" ( $p$. 24). Los didactas Nicole y Fabián comparten la idea de una temporalidad orientada principalmente al compromiso futuro del territorio que implica que los y las estudiantes tengan la posibilidad de pensar en futuros alternativos, probables, posibles y preferibles (Ortega, 2019).

La enseñanza de la Geografía se ha centrado principalmente en los territorios, relegando muchas veces a los hombres y mujeres a un segundo plano. Autores como Fien (1992), Gurevich et al. (1995), Braudel (2002) y Pagès (2011) han resignificado el protagonismo de la sociedad como un tejido vivo y cambiante en los territorios. Para Braudel (2002) la geografía olvida muy a menudo al hombre, lo social, porque continuamente insiste en el espacio físico, invisibilizando a los agentes sociales que están adheridos a él. Pagès (2011) refuerza estas ideas llamando a "conceder mucho más protagonismo a los hombres y a las mujeres que a los territorios" (p.20).

Por su parte, Gurevich et al. (1995) plantean que la relación sociedad - naturaleza es fundamental, y significa tratar en el aula a hombres y mujeres reales. Implica la aparición de agentes sociales donde los y las estudiantes puedan reconocerse. Estos hombres y mujeres, ciudadanos y ciudadanas son quienes toman decisiones territoriales y espaciales importantes para llevar a cabo acciones en el territorio.

\section{Marco Metodológico}

Nuestra investigación se desarrolla en el campo de la Didáctica de las Ciencias Sociales. Está protagonizada por el profesorado de secundaria, y, en menor medida, por el alumnado y los contenidos con la finalidad de observar y comprender, de manera holística, que sucede en las aulas respecto a la formación de la conciencia geográfica. Se ubica en el paradigma constructivista-crítico porque busca analizar la realidad para transformarla (Pagès \& Santisteban, 2011; Cohen, Manion \& Morrison, 2007).

Se aplica una metodología mixta con predominio del enfoque cualitativo a partir de un estudio de casos según los planteamientos de Stake (1999) y Simons (2011). El estudio de caso es una metodología especialmente apropiada para analizar problemas de la práctica educativa (Simons, 2011). En esta investigación se estudian el pensamiento y las prácticas de un grupo de docentes en ejercicio de tres colegios de la Región de la Araucanía, Chile.

El valor de una investigación educativa centrada en el docente radica en que su finalidad es ayudar al profesorado de Geografía a iniciar un proceso de análisis y reflexión de su práctica que podrá conducirle a su mejora.

Para los fines de la investigación se aplican diversos instrumentos de recogida de información: análisis documental del currículo, cuestionario (21 participantes), consulta online a un grupo de didactas en el área de Ciencias Sociales de diferentes países (Chile, España, Argentina, Brasil, Portugal, Inglaterra, Francia y Canadá), entrevistas al profesorado y observaciones de aula (5 participantes) y grupos focales con el estudiantado. 
En este artículo, presentaremos los resultados de las observaciones, cuyas interpretaciones se realizaron a partir de los ejes orientadores propuestos para abordar la conciencia geográfica en el aula. Se realizaron observaciones no participantes en el período comprendido entre el 12 de abril y el 27 de abril de 2018. Se observaron tres clases (90 minutos cada una) de cada docente, a excepción de Marta, de quien sólo fue posible observar dos clases. El registro de lo observado se realizó a través de notas de campo y acompañadas de la grabación en audio.

La observación de las clases se produjo durante un periodo escolar en el que la enseñanza se centró principalmente en contenidos de carácter histórico. Sin embargo, pudimos relacionarlos con relativa facilidad con la perspectiva geográfica de los y las docentes.

Se ha respetado el anonimato de los y las docentes a través de seudónimos que hemos asignado en el cuestionario, las entrevistas y las observaciones. El cuadro $\mathrm{N}^{\circ} 2$ muestra algunas características de los y las docentes participantes. La selección del profesorado fue intencional, según la cercanía para el trabajo de campo y la buena predisposición de los y las docentes de colaborar en la investigación.

Cuadro $\mathrm{N}^{\circ} 2$.

Características de los y las docentes participantes en las observaciones de aula

\begin{tabular}{|c|c|c|c|c|c|c|c|}
\hline Docente & Edad & $\begin{array}{c}\text { Experiencia } \\
\text { en aula }\end{array}$ & $\begin{array}{c}\text { Dependencia } \\
\text { colegio }\end{array}$ & $\begin{array}{c}\text { Modalidad } \\
\text { colegio }\end{array}$ & $\begin{array}{c}\text { Ciudad } \\
\text { desempeño }\end{array}$ & Curso & $\mathbf{N}^{\circ}$ Est \\
\hline Nuria & 35 & 12 años & $\mathrm{P} / \mathrm{S}$ & $\mathrm{C} / \mathrm{H}$ & Temuco & 8 vo & 44 \\
\hline Marco & 30 & 7 años & $\mathrm{P} / \mathrm{S}$ & $\mathrm{C} / \mathrm{H}$ & Temuco & 1 ro.M & 38 \\
\hline Pablo & 59 & 36 años & $\mathrm{M}$ & $\mathrm{T} / \mathrm{P}$ & Angol & 1 ro.M & 44 \\
\hline Montserrat & 50 & 27 años & $\mathrm{P} / \mathrm{S}$ & $\mathrm{C} / \mathrm{H}$ & Angol & 2 do.M & 40 \\
\hline Marta & 34 & 11 años & $\mathrm{P} / \mathrm{S}$ & $\mathrm{C} / \mathrm{H}$ & Angol & $8 \mathrm{vo}$ & 37 \\
\hline
\end{tabular}

Fuente: Elaboración propia

Notas: P: Particular; P/S: Particular-Subvencionado; M: Municipal; C/H: Científico Humanista; T/P: Técnico Profesional

\section{Formación de la conciencia geográfica en las prácticas de aula}

En Chile, la asignatura de Historia y Ciencias Sociales está compuesta por un plan de contenidos históricos y geográficos que se organiza en objetivos de aprendizaje a lo largo del curso escolar. El profesorado que participó en la investigación mantiene en sus clases la lógica curricular, es decir, una organización temporal diacrónica (desde la hominización hasta la actualidad) y una secuencia espacial concéntrica (desde lo local a lo global). Esta perspectiva de contenidos hace que el conocimiento que se trata sea, muchas veces, fragmentado y descontextualizado temporal y espacialmente.

En líneas generales, las clases observadas priorizaron temas históricos (sociales - políticos militares - económicos - territoriales) y la perspectiva geográfica aparece principalmente como una herramienta subsidiaria para localizar hechos o países, observar mapas, situar el contexto 
local, nacional o mundial, y explicar las expansiones territoriales. El profesorado trabajó los temas mayoritariamente desde perspectivas expositivas, descriptivas y eurocéntricas, manifestando la enseñanza de un conocimiento geográfico fragmentado.

El cuadro $\mathrm{N}^{\circ} 3$ presenta los resultados sobre los niveles de desarrollo de la conciencia en que se sitúa el profesorado que participa en la investigación según los contenidos tratados, las habilidades y los tres elementos claves (multiescalaridad, temporalidad y protagonistas).

Cuadro $\mathrm{N}^{\circ} 3$.

Niveles de progresión de la conciencia y su tratamiento en el aula

\begin{tabular}{|c|c|c|c|c|c|}
\hline \multirow{3}{*}{\begin{tabular}{c|} 
Nivel Conciencia \\
Docentes \\
Temas
\end{tabular}} & \multicolumn{2}{|c|}{$\begin{array}{l}\text { N1_conciencia no } \\
\text { reflexiva o ingenua }\end{array}$} & \multirow{2}{*}{$\begin{array}{c}\begin{array}{c}\text { N2_conciencia des- } \\
\text { criptiva }\end{array} \\
\text { Montserrat } \\
\end{array}$} & \multicolumn{2}{|c|}{ N3_conciencia analítica } \\
\hline & \multirow{2}{*}{\begin{tabular}{|l|}
\multicolumn{1}{|c|}{ Marta } \\
Absolutis- \\
mo España \\
y Francia
\end{tabular}} & \multirow[b]{2}{*}{$\begin{array}{l}\text { Nuria } \\
\text { Exp a n - } \\
\text { sión eu- } \\
\text { r o p e a } \\
\text { / Con- } \\
\text { quista de } \\
\text { América. }\end{array}$} & & \multirow{2}{*}{\begin{tabular}{|l|}
\multicolumn{2}{|c|}{ Marco } \\
Conformación \\
del Estado - \\
Nación Chile s. \\
XIX
\end{tabular}} & Pablo \\
\hline & & & $\begin{array}{l}\text { Expansión territorial } \\
\text { Chile S. XIX }\end{array}$ & & $\begin{array}{l}\text { Rev. Indus- } \\
\text { trial / Migra- } \\
\text { ciones }\end{array}$ \\
\hline Habilidades & $\begin{array}{l}\text { Desarrollan } \\
\text { des orienta } \\
\text { repetición } \\
\text { zación porq } \\
\text { tran en ir } \\
\text { nes, fechas } \\
\text { definiciones }\end{array}$ & $\begin{array}{l}\text { habilida- } \\
\text { adas a la } \\
\text { y memori- } \\
\text { que se cen- } \\
\text { nformacio- } \\
\text { s, lugares, } \\
\text { s. }\end{array}$ & $\begin{array}{l}\text { Desarrolla habilida- } \\
\text { des de tipo descripti- } \\
\text { vo porque se centran } \\
\text { en conceptos y en } \\
\text { el relato de hechos } \\
\text { geográficos y las ca- } \\
\text { racterísticas espacio } \\
\text { - temporales que les } \\
\text { rodean. }\end{array}$ & \multicolumn{2}{|c|}{$\begin{array}{l}\text { Plantean habilidades de } \\
\text { análisis que van desarrollan- } \\
\text { do en sus clases a través } \\
\text { de preguntas/ respuestas y } \\
\text { ejemplos de la vida cotidiana } \\
\text { (búsqueda de relaciones, la } \\
\text { asociación de hechos, cau- } \\
\text { sas/consecuencias, etc). }\end{array}$} \\
\hline Multiescalaridad & $\begin{array}{l}\text { Localizaciór } \\
\text { tos específi } \\
\text { mapa / Inv } \\
\text { elementos } \\
\text { cos. }\end{array}$ & $\begin{array}{l}n \text { de pun- } \\
\text { icos en un } \\
\text { entario de } \\
\text { geográfi- }\end{array}$ & $\begin{array}{l}\text { Se mueve en escalas } \\
\text { locales y globales en } \\
\text { una relación lineal. }\end{array}$ & \multicolumn{2}{|c|}{$\begin{array}{l}\text { Se mueven y conectan diver } \\
\text { sas escalas (global, local o } \\
\text { viceversa). }\end{array}$} \\
\hline Temporalidad & $\begin{array}{l}\text { Se centran } \\
\text { do (S.XV - > } \\
\text { Monarquías } \\
\text { de Francia ) } \\
\text { la expansiór }\end{array}$ & $\begin{array}{l}\text { en el pasa- } \\
\text { XVI) en las } \\
\text { absolutas } \\
\text { y España y } \\
\text { n europea. }\end{array}$ & $\begin{array}{l}\text { Trata la temporalidad } \\
\text { desde el pasado al pre- } \\
\text { sente (Ej. migraciones: } \\
\text { Guerra del Pacifico y } \\
\text { actualidad), centrán- } \\
\text { dose en el pasado. }\end{array}$ & \multicolumn{2}{|c|}{$\begin{array}{l}\text { Analizan los contenidos des } \\
\text { de una mirada temporal dia- } \\
\text { crónica (pasado - presente). }\end{array}$} \\
\hline Protagonistas & \multicolumn{2}{|c|}{$\begin{array}{l}\text { Los protagonistas son } \\
\text { casi invisibilizados, } \\
\text { aunque en el relato } \\
\text { aparecen en ocasio- } \\
\text { nes hombres blancos, } \\
\text { europeos y con poder } \\
\text { político (reyes, con- } \\
\text { quistadores). }\end{array}$} & $\begin{array}{l}\text { Se centra en territorios } \\
\text { en disputa o en pun- } \\
\text { tos estratégicos para } \\
\text { la economía, donde } \\
\text { los hombres y muje- } \\
\text { res son invisibilizados } \\
\text { exceptuando hombres } \\
\text { con poder político } \\
\text { (Presidentes de la Re- } \\
\text { pública). Y, en una de } \\
\text { las clases se trata el } \\
\text { pueblo originario se- } \\
\text { lknam. }\end{array}$ & \multicolumn{2}{|c|}{$\begin{array}{l}\text { Marco se centra en los terri- } \\
\text { torios en disputa y las rela- } \\
\text { ciones con las personas que } \\
\text { los habitan. Ej. Situación de } \\
\text { pueblo mapuche en Chile. } \\
\text { Pablo se centra en los mi- } \\
\text { grantes (hombres, mujeres, } \\
\text { niños, niñas, jóvenes y ancia- } \\
\text { nos) en los que sus estudian- } \\
\text { tes se ven reflejados porque } \\
\text { plantea preguntas asociadas } \\
\text { a su realidad (amigos y/o fa- } \\
\text { miliares migrantes). }\end{array}$} \\
\hline
\end{tabular}


Estos son dos ejemplos de las actividades realizadas en sus clases por Marta (situada en el N1_conciencia no reflexiva) y Pablo (situado en el N3_conciencia analítica).

El tema que desarrolló Marta era el Absolutismo en España y Francia. En el diario de campo anotamos lo siguiente:

Marta entrega el mapa "Europa en el siglo XVI". La docente solicita que localicen y pinten los diferentes territorios de los gobiernos en Europa en el siglo XVI. Proyecta el mapa en la pizarra. Luego dibuja la simbología en la pizarra y modifica los colores pre-existentes en el mapa que se encuentra proyectado. Marta pide a los estudiantes que en color amarillo pinten los territorios de las monarquías absolutas, en verde las ciudades estado italianas y la monarquía parlamentaria inglesa y en rojo el sacro imperio romano. Un grupo importante de estudiantes copian el mapa que muestra la docente en la pizarra y no modifican los colores. La actividad tiene una duración de 10 minutos.

(notas de campo, 27 de abril de 2018).

Figura $\mathrm{N}^{\circ} 2$.

Pizarra sala de clases (objetivo de la clase, mapamundi y proyección del mapa de Europa en el siglo $\mathrm{XVI}$ ).

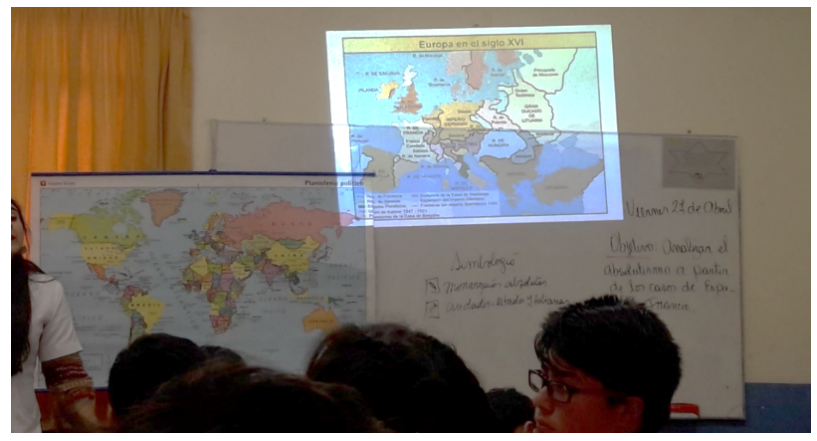

Fuente: Diario de campo, 27 de abril de 2018.

Figura $\mathrm{N}^{\circ} 3$.

Cuaderno Alumna F (actividad mapa "Europa en el s. XVI")

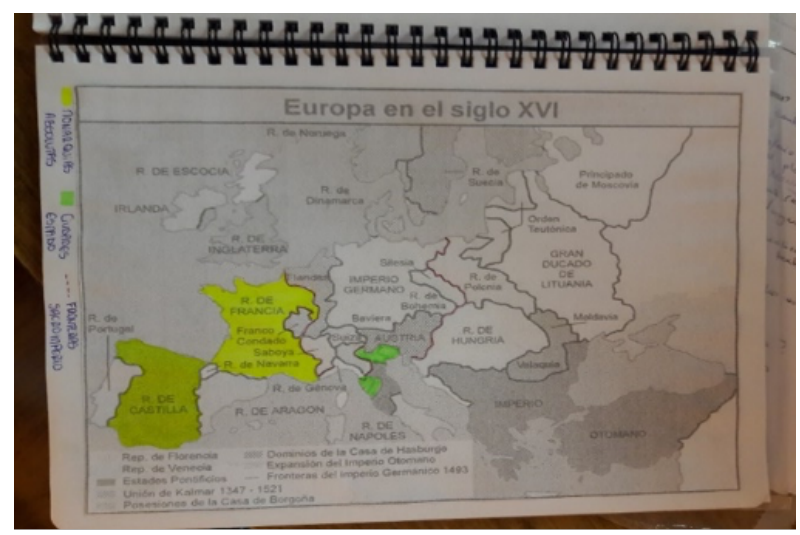

Fuente: Diario de campo, 27 de abril de 2018. 
Marta desarrolla una conciencia geográfica no reflexiva porque propone actividades de carácter repetitivo y memorístico. Utiliza el mapa como recurso auxiliar de los contenidos históricos, es decir, presenta el territorio como un soporte donde tienen lugar las actividades humanas. Se centra exclusivamente en la localización.

El mapa ofrece una narrativa cartográfica que va más allá de la mera localización. Marta podría haber considerado, además, las diversas realidades políticas, económicas, sociales y ambientales que involucran las relaciones de diferentes grupos que viven e interactúan de diferentes maneras en esas realidades. Los mapas no son solo la representación de un espacio contenedor, sino que son representaciones de un espacio vivo, construido históricamente y que, en muchas ocasiones, se utiliza como herramienta de poder.

Pablo, por su parte, trabajó la Revolución Industrial y las migraciones. Esto es lo que anotamos en el diario de campo:

El docente plantea una situación hipotética para introducir los contenidos de la clase.

Pablo solicita a los estudiantes que registren la siguiente pregunta: Si usted tuviera que emigrar a otro país ¿a dónde lo haría? ¿Cuál sería? ¿Por qué ese país? ¿Qué inconvenientes encontraría?

Alumna 5: Yo me iría a África para trabajar con los niños pobres. El inconveniente sería el idioma y el hospedaje. También enfermedades.

Alumna 9: a mí me gustaría conocer Estados Unidos porque me gusta las costumbres, las personas. Inconvenientes el idioma.

(notas de campo, 18 de abril de 2018).

Pablo se sitúa en el nivel de la conciencia analítica en sus clases porque realiza constantes preguntas de tipo abiertas e inductivas. Son preguntas que se relacionan con la vida cotidiana y con las experiencias sociales y espaciales del estudiantado. Desde temáticas como la Revolución Industrial (tema explícito en el currículo), el docente lleva a sus estudiantes a problemas actuales, importantes, urgentes, que suscitan el interés y que sitúan al estudiantado ante las migraciones actuales en Chile (tema no explícito en el currículo). En el ejemplo, el docente permite a los estudiantes posicionarse en el papel de una persona que migra, tomar decisiones y argumentarlas.

El profesor se mueve por, y conecta, diversas escalas (global, local o viceversa), analiza los contenidos desde una mirada temporal diacrónica (pasado - presente) y se centra en los migrantes (hombres, mujeres, niños, niñas, jóvenes y ancianos) en los que sus estudiantes se ven reflejados porque plantea preguntas asociadas a su realidad (amigos y/o familiares migrantes).

Observamos que ningún docente alcanza el desarrollo de un N_4 de conciencia crítica en sus clases, porque no llegan a establecer directrices de aplicación o acción en los contenidos geográficos tratados, no plantean actividades donde los estudiantes se asuman como ciudadanas y ciudadanos partícipes y activos en su realidad geográfica y con ello se sientan responsables de los problemas de su entorno. El profesorado de nuestra investigación no orienta en las actividades a sus estudiantes a pensar en alternativas a los problemas geográficos y/o ambientales de su realidad. 


\section{Conclusiones}

Una de las dificultades con las que se encuentra este profesorado es la de situarse frente a los contenidos geográficos de manera descontextualizada y fragmentada respecto al resto de contenidos históricos y sociales. Para llegar a la formación de una conciencia geográfica crítica es necesario y urgente posicionarse en una enseñanza de la geografía centrada en problemas sociales relevantes. A partir del estudio de problemas el profesorado podrá ahondar en situaciones importantes y vivenciales para la sociedad apostando por la formación de una conciencia geográfica en su alumnado que se active tanto individual como colectivamente.

En este sentido, cuando el profesorado enseña contenidos a partir de problemas geográficos y ambientales relevantes capacita a sus estudiantes para analizar y valorar evidencias, tomar decisiones fundamentadas, resolver problemas cotidianos de sus vidas y desarrollar una fundamentación ética de sus relaciones personales y sociales (Fien, 1992; Benejam, 2003; Pagès, 2007; Pagés \& Santisteban, 2011).

Estas ideas son compartidas por diversos autores (por ejemplo, Fien, 1992; Merenne - Schoumaker, 2005; Le Roux, 1997; Souto, 2007a, 2007b; García, 2011; Cavalcanti \& De Souza, 2015), por algunas propuestas curriculares (Australia, Nueva Zelanda, Quebec, Singapur) y por los y las Didactas de las Ciencias Sociales entrevistados en la investigación (Ortega, 2019).

Creemos que los resultados de esta investigación son un reto tanto para los docentes como para las Universidades en las que se forman, que deberían centrarse más la preparación didáctica tanto inicial como continua del profesorado chileno. Una preparación enfocada en la formación de un pensamiento y una conciencia geográfica crítica a fin de que la ciudadanía pueda hacer frente a los retos actuales y futuros de su territorio y del mundo.

\section{Referencias}

ARAYA, F. Formación Ciudadana desde la Educación Geográfica. La Serena: Universidad de la Serena, 2013.

ARENAS, A. Conclusiones relevantes de investigaciones chilenas en Educación Geográfica. En: CÂMARA, A.; SANDE E. y MAGRO, M. (eds.). Atas do VIII Congresso Ibérico de Didática da Geografia. Educação Geográfica na Modernidade Líquida. Lisboa: Associação de Professores de Geografia, 2017, p. 677-692.

ARENAS, A. \& SALINAS, V. Giros en la Educación Geográfica: renovación de lo geográfico y lo educativo. Revista de Geografía del Norte Grande, 2013, № 56, p. 143-162. https://dx.doi.org/10.4067/ S0718-34022013000300008

ARENAS, A.; FERNÁNDEZ, H. \& PÉREZ, P. (eds.). Una Educación Geográfica para Chile. Santiago de Chile: Sociedad Chilena de Ciencias Geográficas, 2016. [Consulta: 02-06-2020]. Disponible 
en: http://sociedadchilenadecienciasgeograficas.cl/2014/wp-content/uploads/2016/11/SOCHIGEO-2016-UNA-EDUCACION-GEOGRAFICA-PARA-CHILE.pdf

BAHBAHANI, K., DUQUETTE, C., SHARPE, B., \& TU HUYNH, N. Enseigner la pensée géographique. Vancouver: The Critical Thinking Consortium, 2016.

BENEJAM, P. La didáctica de la geografía desde la perspectiva constructivista. Documents D 'Análisi Geográfica, 1992, № 21, p. 35 -52.

BENEJAM, P. Las finalidades de la educación social. En: BENEJAM, P. y PAGĖS, J. (eds.). Enseñar y aprender Ciencias Sociales, Geografía e Historia en la Educación Secundaria. Barcelona: ICE Horsori, 1997a, p. 33-50.

BENEJAM, P. La selección y secuenciación de los contenidos sociales. En: BENEJAM, P. y PAGĖS, J. (eds.). Enseñar y aprender Ciencias Sociales, Geografía e Historia en la Educación Secundaria. Barcelona: ICE Horsori, 1997b, p. 71-96.

BENEJAM, P. La enseñanza de la Geografía. Problemas y propuestas en la sociedad actual. En: MARRÓN, M.; MORALEDA, C. \& RODRÍGUEZ, H. (eds.). La enseñanza de la geografía ante las nuevas demandas sociales. Toledo: AGE - Universidad Castilla - La Mancha, 2003, p. 551 - 562.

BENEJAM, P. Conceptes bàsics sobre l'espai en l'ensenyança i aprenentatge de la geografía. Perspectiva escolar, 2011, N 358, p. $2-13$.

BRAUDEL, F. Las ambiciones de la Historia. Barcelona: Crítica, 2002.

CALLAI, H. Educação geográfica: ensinar e aprender Geografía. En: CASTELLAR, S. y MUNHOZ, G. (eds.). Conhecimentos escolares e caminhos metodológicos. São Paulo: EJR Xamã, 2012, p. $73-88$.

CAVALCANTI, L. A geografía escolar e a cidade: ensaios sobre o ensino de Geografía para a vida urbana cotidiana. Campinas: Papirus, 2008.

CAVALCANTI, L. Geografia escolar, formação e práticas docentes: percursos trilhados. En: CASTELLAR, S. \& MUNHOZ, G. (eds.). Conhecimentos escolares e caminhos metodológicos. São Paulo: EJR Xamã Editoria, 2012, p. $89-100$.

CAVALCANTI, L. \& DE SOUZA, V. (2015). Experiencias escolares de Enseñanza de la Geografía: estrategias para hacer visible al joven ciudadano. En: HERNÁNDEZ, A.; GARCÍA, C. \& DE LA MONTAÑA, J. (eds.). Una enseñanza de las ciencias sociales para el futuro: recursos para trabajar la invisibilidad de personas, lugares y temáticas. Cáceres: Asociación Universitaria del Profesorado en Didáctica de las Ciencias Sociales, 2015, p. 987 - 993.

COHEN, L.; MANION, L. \& MORRISON, K. Research Methods in Education. London: Routledge, 2007. 
COMES, P. El espacio en la didáctica de las ciencias sociales. En: TREPAT, C. y COMES, P. (eds.). El tiempo y el espacio en la didáctica de las ciencias sociales. Barcelona: Graó, 1998, p. 123- 190.

FERNÁNDEZ, M. Geografía y Formación Ciudadana en el nuevo milenio: elementos para una transmisión significativa de contenidos escolares. Diez años de cambios en el Mundo, en la Geografía y en las Ciencias Sociales, 1999-2008. En: X Coloquio Internacional de Geocrítica, 26 a 30 de mayo [en línea]. Universidad de Barcelona, 2008. [Consulta: 02-06-2020]. Disponible en: http://www. ub.es/geocrit/-xcol/196.htm

FREIRE, P. La educación como práctica de la libertad. Montevideo: Siglo XXI Argentina Editores, 1980.

FIEN, J. Geografía, sociedad y vida cotidiana. Documents D’Análisi Geográfica, 1992, № 23, p. 73-90.

FIEN, J. Towards a map of commitment: A socially critical approach to Geographical Education. Internacional Research in Geographical and Environmental Education, 1999, № 8, p. 140-158.

GARCÍA, F. Problemas del mundo y educación escolar: un desafío para la enseñanza de la geografía y las ciencias sociales. Revista Brasileira de Educação em Geografia, 2011, № 1, p. 108 - 122.

GARCÍA, F. Ciudadanía Participativa y trabajo en torno a problemas sociales y ambientales. En: PAGĖS, J. \& SANTISTEBAN, A. (eds.). Una mirada al pasado y un proyecto de futuro. Investigación e innovación en didáctica de las ciencias sociales. Barcelona: Asociación Universitaria del Profesorado en Didáctica de las Ciencias Sociales, 2014, p. 119 - 125.

GARDNER, R. \& LAMBERT, D. Futuring Geographers: The role of the subject organisations. Geography, 2006, N 91, p. 159-170. https://doi.org/10.1080/00167487.2006.12094161

GARRIDO, M. El espacio por aprender, el mismo que enseñar: las urgencias de la educación geográfica. Cedes, 2005, №66, p. 137-163. https://doi.org/10.1590/S0101-32622005000200002

GUREVICH, R., BLANCO, J., FERNÁNDEZ, M. \& TOBÍO, O. Notas sobre la enseñanza de una geografía renovada. Buenos Aires: Aique, 1995.

HICKS, D. Lessons for the Future a geographical contribution. Geography, 2007, № 92, p. 179-188. https://doi.org/10.1080/00167487.2007.12094198

HICKS, D. A geography of hope. Geography, 2014, N 99, p. 5-12. https://doi.org/10.1080/001674 87.2014.12094385

HIGUERAS, A. Teoría y método de la Geografía. Introducción al análisis geográfico regional. Zaragoza: Prensas Universitarias de Zaragoza, 2003.

KLEIN, J. \& LAURIN, S. L'education géographique. Formation du citoyen et conscience territoriale. Québec: Presses de l'Université du Québec, 1999. 
LAURIN, S. Éduquer à la pensée en géographie scolaire: cerner ce quelque chose de fondamental. En: GOHIER, C. \& LAURIN, S. (eds.). Entre culture, compétence et contenu: la formation fondamentale, un espace à redéfinir. Québec: Les Éditions Logiques, 2001, p. 195-228.

LEFEBVRE, H. La producción del espacio. Madrid: Capitán Swing, 2013.

LE ROUX, A. Didactique de la géographie. France: Presses Universitaires de Caen, 1997.

MATTOZZI, I. ¿Quién tiene miedo de la Geohistoria? Enseñanza de las Ciencias Sociales, 2014, № 13, p. $85-105$.

MERENNE - SCHOUMAKER, B. Didactique de la géographie. Organiser les apprentissages. BruxeIles: De Boeck y Larcier, 2005.

MÉVEL, Y. \& TUTIAUX - GUILLON, N. Didactique et enseignement de I'historie- géographie au collège et au lycée. París: EPU, 2013.

MIRANDA, P. La educación geográfica en Chile: desde su aparición en el currículum escolar en el Siglo XIX hasta los ajustes curriculares de 2010. Anekumene, 2012, № 4, p. 51-71.

ORTEGA, E. La conciencia geográfica. Reflexiones y aportes desde la Didáctica de las Ciencias Sociales. En: MARTíNEZ, P. y GARÍN, A. (eds.). Medio siglo de Geografías en la Araucanía. Temuco: Ediciones Universidad de la Frontera, 2019, p. 277 - 292.

ORTEGA, E. Concepciones del profesorado chileno sobre la conciencia geográfica. Revista de Investigación en Didáctica de las Ciencias Sociales, 2020, №6, p. 126-134. https://doi. org/10.17398/2531-0968.06.126

PAGÈS, J. Los valores y la didáctica de las Ciencias Sociales: retos para la formación de una conciencia democrática. En: FILELLA, A. (ed.). Los valores y la didáctica de las Ciencias Sociales. Lleida: Asociación Universitaria del Profesorado en Didáctica de las Ciencias Sociales, 1998, p. 7-20.

PAGĖS, J. La enseñanza de las Ciencias Sociales y la educación para la ciudadanía en España. Revista Didáctica Geográfica, 2007, № 9, p. 205 - 214.

PAGĖS, J. Enseñar y aprender ciencias sociales en el siglo XXI: reflexiones casi al final de una década. En: Investigación en Educación, Pedagogía y Formación Docente, II Congreso Internacional. Libro 2, Medellín. Universidad pedagógica nacional, Universidad de Antioquia, Corporación interuniversitaria de servicios, 2009, p. 140-154.

PAGĖS, J. Preguntas, problemas y alternativas para la enseñanza de las Ciencias Sociales en el siglo XXI. Cuadernos de México, 2009, N 1, p. 39 - 53. [Consulta: 03-09-2020]. Disponible en: https://sede.educacion.gob.es/publiventa/descarga.action?f_codigo_agc=15211. 
PAGĖS, J. Educación, ciudadanía y enseñanza de la historia. En: GUIMÂRAES, S. \& GATTI, D.(eds.). Perspectivas do ensino de história: enseino, cidadania e consciência histórica. Uberlândia: EDUFU/ FAPEMIG, 2011, p. 17-31

PAGĖS, J. \& SANTISTEBAN, A. La investigación sobre la enseñanza y el aprendizaje de las Ciencias Sociales en Educación Primaria. En: SANTISTEBAN, A. \& PAGĖS, J. (eds.). Didáctica del conocimiento del medio social y cultural en la educación primaria: ciencias sociales para comprender, pensar y actuar. Madrid: Síntesis, 2011, p. 105 - 121.

ROMERO, J. \& GÓMEZ, A. El conocimiento socio-geográfico en la escuela: las tensiones inherentes a la transmisión institucionalizada de cultura y los dilemas de la educación para la democracia en este mundo globalizado. Scripta Nova. Revista Electrónica de Geografía y Ciencias Sociales, 2008, Vol. XII, N² 270. [Consulta: 25-06-2020]. Disponible en: http://revistes.ub.edu/index.php/ ScriptaNova/article/view/1546.

SANTISTEBAN, A. La formación de competências de pensamiento histórico. Clio y asociados, 2010, No 14, p. 34-56.

SANTOS, M. Por una Geografía nueva. Madrid: Espasa Calpe, 1990.

SIMONS, H. El estudio de caso: Teoría y práctica. Madrid: Morata, 2011.

SOUTO, X. Geografía y ciudadanía. Espacio público educativo y geografía escolar: los retos para una formación ciudadana. En: ÁVILA, R.; LÓPEZ, R. \& FERNÁNDEZ, E. (eds.). Las competencias profesionales para la enseñanza aprendizaje de las Ciencias Sociales ante el reto europeo y la globalización. Bilbao: Asociación Universitaria de Profesores de Didáctica de las Ciencias SocialesUniversidad del País Vasco, 2007a, p. 217 - 240.

SOUTO, X. Educación Geográfica y Ciudadanía. Didáctica de la Geografía, 2007b, №9, p. 11-32.

STAKE, R. E. Investigación con estudio de casos. Madrid: Morata, 1999.

STOPANI, R. La ricerca storico-territoriale. Firenze: Editrice Fiorentina, 1978.

TUTIAUX-GUILLON, N. Los fundamentos de una investigación sobre la concepción de las finalidades cívicas y culturales del profesorado de geografía e historia. Enseñanza de las ciencias sociales, 2003, № 2, p. 27-35.

TUTIAUX - GUILLON, N. Las investigaciones en Didáctica sobre la clase de Historia o de Geografía: Balances y reflexiones. En: GÓMEZ, A. \& NÚÑEZ, M. (eds.). Formar para Investigar, Investigar para Formar en Didáctica de las Ciencias Sociales. Málaga: Asociación Universitaria de Profesores de Didáctica de las Ciencias Sociales, 2006, p. 19-38. 
TUTIAUX-GUILLON, N. Interpréter la stabilité d'une discipline scolaire: I'histoire-géographie dans le secondaire français. En: AUDIGIER, F. \& TUTIAUX-GUILLON, N. (eds.). Compétences et contenus. Les curriculums en questions. Bruxelles: De Boeck, 2008, p. 117-146.

UGI. Declaración Internacional sobre Educación Geográfica, Unión Geográfica Internacional, Pekín, 2016. 